\title{
Optikron: A Tool Suite for Enhancing Model-Checking of Real-Time Systems
}

\author{
Conrado Daws * \\ VERIMAG \\ Centre Équation, 2 avenue de Vignate, 38610 Gières, France.
}

\begin{abstract}
OPTIKRON is a tool suite implementing a set of efficient techniques which can be combined to reduce the number of clock variables of a timed system specification without changing its behavior. Thus, the performance of the model-checking procedures can be improved, both in memory space and running time, as we show for the reachability analysis.
\end{abstract}

\section{Introduction}

The most commonly used techniques for the automatic verification of real-time systems $[2,6]$, based on model-checking, suffer from the state-space explosion problem. That is, their cost grows drastically not only with the number of components, as in the untimed case, but also with the number of clock variables used in the specification of the system $[1,3,5]$.

OPTIKRON offers a set of efficient techniques that can be combined to reduce the number of clocks of a timed system specification without changing its behavior. Using OptIKRoN as the first step of the verification process can improve the performance of the model-checking procedures of the verification tools, whenever a global reduction of the number of clocks is possible, that is, a model with the same behavior and less clocks can be found.

Moreover, even when there is no global reduction, local reductions are always important, because all of the clocks are not needed for the representation of the time constraints of a timed system. OptIKRon shows how an encoding of the clock-space based on the local reduction results always lead to an important gain, both in time and space, for the computation of the set of reachable states of the system. The efficiency of the model-checking procedures should also be improved if they take into account the local reduction information.

To our knowledge, this is the first succesfull attempt in applying techniques of variable optimization to the case of real-time systems. Related work can be found in [7] where the problem of minimization of timed systems is studied from a theoretical point of view, and in [5] where linear relations between clocks are detected while computing the set of reachable states, with a more expensive algorithm than ours.

* wW: http://ww-verimag.imag.fr/PEDPLE/Conrado.Daws/ 


\section{Clock Reduction Techniques}

The main clock-reduction techniques implemented in OPTIKRon, namely the activity and the equality reduction, are techniques of static analysis of real-time systems based on fix-point computations [4].

The first one consists in detecting active clocks, that is, clocks that are relevant to the behavior of the system in a given control location. Thus, inactive clocks can be removed without affecting the system behavior. The second one consists in detecting clocks that are always equal in a given location. Thus, they can be collapsed into a unique clock without loss of information ${ }^{1}$. Furthermore, since the activity reduction and the equality reduction are orthogonal, they can be combined and their combination can lead to a better reduction.

\section{Verification}

Although Optikron can not be considered a verification tool on its own, its main goal is to provide useful information on the clock variables of the model a system, in order to reduce the size of the clock-space and, thus, improve the efficiency of the real-time model-checking procedures implemented in tools like KRONOS [2] and UPPAAL [6].

Moreover, OPTIKRON can compute more efficiently the set of reachable states of a real-time system optimizing the size of the clock-space with respect to the results on the local clock-reductions. This can be useful enough in order to verify safety properties of the system.

\section{Experimental Results}

Table 1 shows the results of the application of the reduction techniques of OpTIKRON to several case studies of real-time systems, coming from a wide range of application areas: communication protocols (FDDI, CSMA-CD, Fischer, TickTock), control systems (PLANT, MODUL, ROBOT), and circuits (MOS).

The first three columns of the table give the size of the timed automaton modeling the example. Central columns give the clock-reduction obtained with OptIKRon(number of iterations and clocks) by applying just the activity or the equality reduction technique, and by combining both of them. Last column shows the memory saving by representing the time constraints of the timed automaton with a data structure of variable size based on the local clock-reduction information.

It is worth noticing that even when there is no global reduction, as for CSMA, FISC, and MODUL, the memory saving obtained with the time constraints representation with variable size is really important (from $45 \%$ to $95 \%$ ).

\footnotetext{
${ }^{1}$ Although the algorithm for detecting equal clocks has recently been adapted to cope with general linear relations between clocks, it turns out that in practise the only linear relation between clocks are equality relations.
} 
Table 1. Clock-reduction and memory saving

\begin{tabular}{|c|c|c|c|c|c|c|c|c|c|}
\hline & \multicolumn{3}{|c|}{ Automaton } & \multicolumn{2}{|c|}{ Activity } & \multicolumn{2}{|c|}{ Equality } & Comb. & Saving \\
\hline Example & loc & trans & clo & iter & clo & iter & clo & clo & $\% \mathrm{Mem}$ \\
\hline FDDI 50 & 150 & 200 & 101 & 100 & 51 & 3 & 101 & 51 & 74.2 \\
\hline CSMA 5 & 918 & 4590 & 6 & 2 & 6 & 2 & 6 & 6 & 46.8 \\
\hline FISC 5 & 2402 & 5850 & 5 & 2 & 5 & 2 & 5 & 5 & 79.2 \\
\hline TICK & 72 & 240 & 6 & 1 & 5 & 2 & 6 & 4 & 70.0 \\
\hline PLANT & 236 & 602 & 5 & 1 & 4 & 2 & 5 & 4 & 64.2 \\
\hline$\overline{\mathrm{MOS}}$ & 769 & 2683 & 12 & 1 & 10 & 2 & 11 & 6 & 87.5 \\
\hline MODUL & 1421 & 5809 & 5 & 1 & 5 & 2 & 5 & 5 & 59.6 \\
\hline$\overline{\mathrm{ROBOT}}$ & 106 & 12816 & 11 & 1 & 5 & 3 & 6 & 2 & 95.2 \\
\hline
\end{tabular}

\section{Tool Architecture and Distribution}

OPTIKRON consists of a module implementing the clock-reduction techniques, optikron, and a module for computing the set of reachable states of the system, simulakro, with a representation of variable size of the time constraints. Figure 1 presents the architecture of the tool.

\section{The optikron module}

The current version of the optikron module takes as input a timed system specified as a timed automaton in the timed graph format of KRoNOS (.tg files) ${ }^{2}$. The output of this module is a binary file .opt containing the information on the local clock-reductions.

Additional information on these reductions can be obtained, by specifying the corresponding options, in the output text files . act (for the list of the set of active clocks in each automaton location), . equ (for the list of equal clocks in each location), and .I in (for the list of the linear relations between clocks in each location).

The clock reduction of the automaton is obtained by renaming its clocks. The local renamings (mappings from the set of original clocks to a set of new clocks) are given in the .ren file. The corresponding renamed timed automaton can be obtained in the file .opt.tg. Clearly, whenever a global reduction is possible, the verification of this model will be more efficient.

\section{The simulakro module}

The module for computing the set of reachable states takes as input the .opt.tg file containing the global reduction of the system and the .opt file containing the local clock-reduction information, previously computed. The output is the text file .reach containing a representation of the reachable states.

${ }^{2}$ An extension for handling files in the UPPAAL format of timed automata (.ta files) is currently in work. 


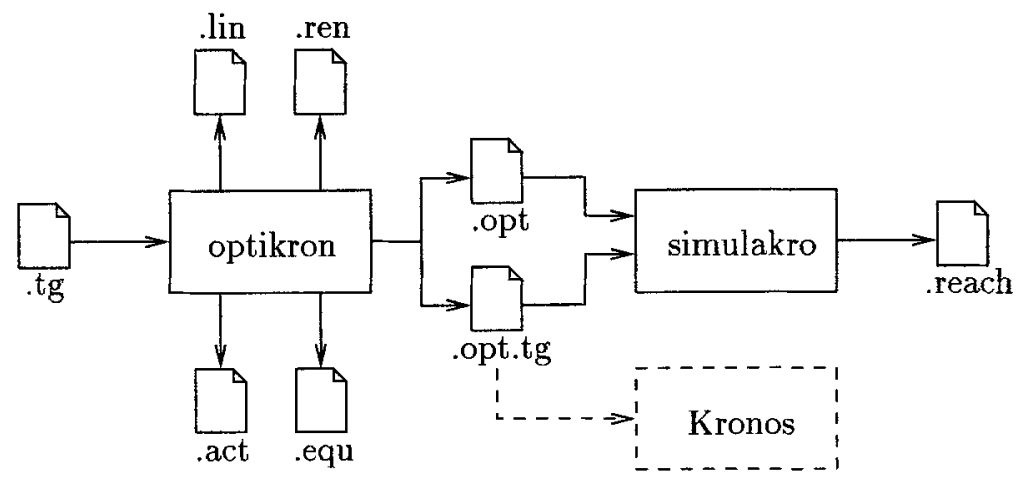

Fig. 1. OptIKRon 2.2 architecture

\section{Availability}

A prototype of Optikron (version 2.2) is freely available for universities and any other non-profit organisms. The distribution package includes executables and a man page. Instructions on how to obtain it, as weel as some additional information on the tool, can be found through the Optikron web page ${ }^{3}$.

\section{References}

1. R. Alur and D.L. Dill. A theory of timed automata. Theoretical Computer Science, 126:183-235, 1994.

2. C. Daws, A. Olivero, S. Tripakis, and S. Yovine. The tool KRONOS. In Hybrid Systems III, Verification and Control, pages 208-219. Lecture Notes in Computer Science 1066, Springer-Verlag, 1996.

3. C. Daws and S. Tripakis. Model-checking of real-time reachability properties using abstractions. In B. Steffen, editor, Proc. of TACAS'98, Lisbon, Portugal, 31 march - 2 April 1998. Springer Verlag, LNCS 1384.

4. C. Daws and S. Yovine. Reducing the number of clock variables of timed automata. In Proc. 1996 IEEE Real-Time Systems Symposium, RTSS'96, Washington, DC, USA, December 1996. IEEE Computer Society Press.

5. K. Larsen, F. Larsson, P. Pettersson, and W. Yi. Efficient verification of real-time systems: Compact data structure and state-space reduction. In Proc. of the 18th IEEE Real-Time Systems Symposium, 1997.

6. K. Larsen, P. Pettersson, and W. Yi. Uppaal: Status \& developments. In Proceedings of $C A V$ '97, Haifa, Israel, 22-25 June 1997.

7. J. Springintveld and F. Vaandrager. Minimizable timed automata. In B. Jonsson and J. Parrow, editors, Proc. of the 4th International Symposium on Formal Techniques in Real Time and Fault Tolerant Systems (FTRTFT'96), volume 1135 of Lecture Notes in Computer Science, pages 130-147, Uppsala, Sweden, 1996. Springer-Verlag.

\footnotetext{
${ }^{3}$ http://www-verimag.imag.fr/PEOPLE/Conrado.Daws/optikron.html
} 